\title{
Estimación de gravedad en cuidados intensivos: validación de índice de estabilidad fisiológica
}

\author{
M.C. Armando Caichac R. ' ; M.C. Pedro Lorca O. ${ }^{2}$; M.C. Sonia Hanning K.' \\ E.U. Sara Per̉a R. ${ }^{3}$; E.U. Maria Neilson V."
}

Severity of illness in intensive care patients: validation of physiologic stability index

Physiological stability index (PSI) is a score proposed to evaluate the severity of different diseases in pediatric intensive care units (PICU) by giving scores to the deterioration of 34 measurable physiological variables. In order to validate PSI, it was prospectively applied to 132 patients who entered our PICU. A significant difference (p $<$ $0.001)$ was found between the average score of those patients who survived $(\bar{x}: 6.1)$ versus those who did not $(\vec{x}: 21)$; No difference was found between theoretical mortality rates obtained by logistic regression analysis and those obtained according to PSI score ranks even thought direct hemodynamic measurements like cardiac output and capillary (wedge) pulmonary pressure were not considered in this study. In conclussion, the PSl is a useful method to measure the severity of illness in pediatric intensive care and to compare results among PICU.

(Key words: intensive care units, severity index, mortality, prognosis.)

Las unidades de cuidados intensivos pediátri$\cos$ atienden pacientes con diferentes diagnósticos y con afecciones de severidad diversa.

Cuando se pretende evaluar el trabajo global de estas unidades o el resultado de diferentes terapias, se encuentran dificultades para controlar el efecto que los distintos niveles de gravedad establecen en las poblaciones en estudio ${ }^{1-4}$.

La necesidad de valorar de manera objetiva la severidad de las enfermedades ha llevado al de. sarrollo de diversos sistemas de medición que han sido validados, demostrando su utilidad en pacientes adultos. ${ }^{5-7}$

Desde el punto de vista pediátrico estos sistemas presentan la desventaja de ser sólo mediciones indirectas de gravedad (TISS) o considerar valores de variables fisiológica propias de la edad adulta (APACHE).

En 1984 Yeh, Pollack $y$ colaboradores elaboraron un sistema de evaluación de gravedad para pacientes pediátricos denominado indice de es. tabilidad fisiológica (IEF), el cual se ha aplicado con buenos resultados en diversas unidades de cuidados intensivos. 8,9

1. Unidad de Cuidados Intensivos Pediátricos, Hospital Dr. Sótero del Río.

2. Médico Becado Pediatría, Pontificia Uníversidad Católica de Chile.

3 Enfermera, Unidad de Cuidados Intensivos Pediátrìcos, Hospital Dr. Sótero del Río.
El objetivo de este estudio fue validar el IEF en una unidad local de cuidados intensivos pediátricos.

\section{MATERIAL Y METODO}

La unidad đe cuidados intensivos pediátrios del hospital Sótero del Río consta de 5 camas y atiende pacientes con afecciones médicas o quirúrgicas, excepto recién nacidos. En ella se dispone de recursos para tratar pacientes en estado crítico con las terapias propias del cuidado intensivo (monitor ía invasiva de presión arterial, presión venosa central, ventilación mecánica, etc.). No se efectúan mediciones de débito cardíaco y variables derivadas.

El IEF analiza 34 variables pertenecientes a 7 sistemas fisiológicos: cardiovascular, respiratorio, neurológico, hematológico, renal, gastrointestinal y metabólico (tabla 1).

Cada variable se subơivide en rangos de anormalidad tomando en cuenta la edad y a estos rangos se les asigna una puntuación arbitraria: 1 punto si la anormalidad causa sólo preocupación; 3 puntos si determina cambios en la terapia, y $S$ puntos si implica riesgo de muerte.

Se confeccionó un formulario con las 34 variables fisiológicas del IEF, el cual se aplicó en forma prospectiva durante los primeros cuatro días a todos los pacientes ingresados entre enero y julio de 1988.

Cada día se registró la calificación correspondiente al valor más anormal de cada una de las variables analizadas; los parámetros no medidos no se consideraron. Se realizó la suma diaria de las calificaciones obteniéndose el promedio para cada paciente al final de los cuatro días. Cuando la permanencia en UCIP fue menor de cuatro días se consideró el promedio de los días observados. 
Tabla 1

Intıce de estabilidad fisiológica

Sistema $1+\quad 3+3+$

Cardiovascular

Lactantes

P.A. sist. ( $\mathrm{mm} \mathrm{H}$ )

Frec. Card. (x min)

Niños Mayores

P.A. sist. (mm Hg)

Frec. Card. $(x \min )$

Cualquier edad

P.A. diast. ( $\mathrm{mm} \mathrm{H}$ )

Indice card.

(L $x \min \times \mathrm{m}^{2}$ )

$C(a-v) 02(m l x \%)$

P.V.C. (mm Hg)

P. cuña (ttm Hg)

\section{Respiratorio}

Lactantes

Frec. Resp. (x min)

$$
50 \text { a } 60
$$

61 a 90

$>90$

apnea

Niños Mayores

Frec. Resp. (x min)

30 a 50

50 a 60

200 a 300

$<30 ; 45-50$

$\mathrm{PaCO}_{2}(\mathrm{~mm} \mathrm{Hg})$

\section{Neurológico}

Glasgow (calif.

P.I.C. (mm Hg)

Convulsiones

Pupilas

\section{Hematológico}

Hemoglobina $(\mathrm{g} / \mathrm{d} \mathrm{l})$

Reto, blancos

( $\mathrm{x} \mathrm{mm}^{3}$ )

Plaquetas

$\left(x \mathrm{~mm}^{3}\right)$

Protrombina

(TTPK)

PDF (ug/ml)
8 a 11

15 a 20

Focales

Isocoria

RFM lento

$\begin{array}{cr}40 \text { a } 54 & <40 \\ >160 & >220 \\ 50 \text { a } 74 & <50 \\ 181 \text { a } 220 & \end{array}$

$\begin{array}{cc}50 \text { a } 64 & <50 \\ >200 & \\ 40 \text { a } 59 & >200 \\ 171 \text { a } 200 & <40\end{array}$

$>110$

1,0 a 1,9

$<1,0$

$>6,5$

$>25$
$>70$

40 a 49

$<200$

51 a 65

$<40$

$>65$

5 a 7

21 a 40

Status GM

Anisocoria

$<5$

$>40$

RFM (-)
3 a 5
$<\quad 3.000$

$>40.000$

$<20.000$

18 a 22

3 a 5.000

$>1,5 \times$ control

$>40$
20 a 50.000

$>1$ millón 


\begin{tabular}{|c|c|c|c|}
\hline Sistema & $1+$ & $2+$ & $3+$ \\
\hline \multicolumn{4}{|l|}{ Renal } \\
\hline $\begin{array}{l}\text { Nitrogeno ureico } \\
\left(\mathrm{mg} \times \%_{0}\right)\end{array}$ & 40 a 100 & $>100$ & \\
\hline $\begin{array}{l}\text { Creatinina } \\
(m g \times \%)\end{array}$ & 2,0 a 10,0 & $>10,0$ & \\
\hline $\begin{array}{l}\text { Débito urinario } \\
\text { (m] / } \mathrm{kg} \times \mathrm{hr} \text { ) }\end{array}$ & 0,5 a 1,0 & $<0,5$ & \\
\hline \multicolumn{4}{|l|}{ Gastrointestinal } \\
\hline $\begin{array}{l}\text { TGO+TGP (JU/1) } \\
\text { Amilasa (U/l) } \\
\text { Bilirrubina toł. } \\
\text { (mg } x \% \text { ) } \\
\text { Albúmina }(\mathrm{g} / \mathrm{d} 1)\end{array}$ & $\begin{array}{c}>100 \\
>500 \\
>3,5 \\
1,2 \text { a } 2,0\end{array}$ & & \\
\hline \multicolumn{4}{|l|}{ Metabólico } \\
\hline Sodio (mEq/I) & $\begin{array}{l}115 \text { a } 125 \\
150 \text { a } 160\end{array}$ & $\langle 115$ a $\rangle 160$ & \\
\hline Potasio (mEq/l) & $\begin{array}{l}3,0 \text { a } 3,5 \\
6,5 \text { a } 7,5\end{array}$ & $\begin{array}{l}2,5 \text { a } 2,9 \\
7,6 \text { a } 8,0\end{array}$ & $\begin{array}{l}<2,5 \\
>8,0\end{array}$ \\
\hline $\begin{array}{l}\text { Calcio (mg/dl) } \\
\text { Glucoma (mg/dl) }\end{array}$ & $\begin{array}{c}7,0 \text { a } 8,0 \\
12,0 \text { a } 15,0 \\
40 \text { a } 60\end{array}$ & $\begin{array}{c}5,0 \text { a } 6,9 \\
>15,0\end{array}$ & $<5,0$ \\
\hline $\begin{array}{l}\text { Osmolaridad } \\
(\mathrm{mOsm} / 1)\end{array}$ & $\begin{array}{l}250 \text { a } 400 \\
320 \text { a } 350\end{array}$ & $\begin{array}{l}>400 \\
>350\end{array}$ & \\
\hline $\mathrm{pH}(\mathrm{U})$ & $\begin{array}{l}7,2 \text { a } 7,3 \\
7,5 \text { a } 7,65\end{array}$ & $\begin{array}{l}7,1 \text { a } 7,19 \\
>7,65\end{array}$ & $<7.1$ \\
\hline $\begin{array}{l}\text { Bicarbonato } \\
\text { (mEq/1) }\end{array}$ & $<16 ;>32$ & & \\
\hline
\end{tabular}

\section{Abreviaciones:}

$\mathrm{C}(\mathrm{a}-4) \mathrm{O}_{2}$ : Diferencia en el contenido arterio venoso de ox igeno.

P.V.C.: Presión venosa central,

P. cuña: Presión enclavada en arterja pulmonar.

\section{P.I.C. : Presión intracraneana.}

PDF: Productos de degradación del fíbrinógeno TGO: Transaminasa glutámico oxalacética.

TGP: Transamina ghutámico pirúvica.
Con los datos obtenidos se confeccionó una curva de probabilidad de muerte mediante análisis de regresión logística, la cual se comparó con la mortalidad observada según rangos de calificación. ${ }^{10}$

Los análisis estad ísticos se realizaron mediante prueba de Chi cuadrado de bondad de ajuste; se consideró significativo un p menor de 0,01 .

\section{RESULTADOS}

Las caracter isticas de la población estudiada se pueden observar en la tabla 2 . Ingresaron al estudio 132 pacientes, 81 hombres y 51 mujeres. La edad fluctuó entre 1 y 180 meses, con una mediana de 11 meses. La letalidad observada fue $18,9 \%$.
Lat calitìcación del IEF para los niffos fallecidos fue 21 puntos y 6,1 puntos para los sobrevivientes, diferencia estad isticamente significativa (tabla 3).

\section{Tabla 2}

Características de la población estudiada

$\begin{array}{lc}\text { Ingresos } & 132 \\ \text { Varones } & 81 \\ \text { Mujeres } & 51 \\ \text { Edad (meses) } & \\ \quad \text { Rango } & 1-180 \\ \quad \text { Mediana } & 11 \\ \text { Letalidad } & 25(18,9 \%)\end{array}$


Tabla 3

Sobrevida según valores promedio del indice de estabilidad fisiológica (IEF)

\begin{tabular}{lcc}
\hline $\begin{array}{l}\text { Sobrevida } \\
(n)\end{array}$ & \multicolumn{2}{c}{ IEF } \\
\hline Viros $(107)$ & $* 6,1$ & $($ Rangos) \\
Fallecidos (25) & $* 21,0$ & $(8,0-19)$ \\
\hline
\end{tabular}

$* p<0,0001$

Tabla 4

Sobrevida según rangos de índice de estabilidad fisiológica (lEF)

\begin{tabular}{|c|c|c|c|c|}
\hline \multirow[t]{2}{*}{ Puntaje } & \multicolumn{2}{|c|}{ Vive } & \multicolumn{2}{|c|}{ Fallece } \\
\hline & $\mathbf{n}$ & $\%$ & $\mathrm{n}$ & $\%$ \\
\hline $0-5$ & 49 & 100,0 & 0 & - \\
\hline $5,1-10$ & 40 & 95,2 & 2 & 4,8 \\
\hline $10,1-15$ & 14 & 66,7 & 7 & 33,3 \\
\hline $15,1-20$ & 4 & 57,1 & 3 & 42,9 \\
\hline $20,1-25$ & 0 & - & 6 & 100,0 \\
\hline $25,1-30$ & 0 & - & 3 & 100.0 \\
\hline $30,1 y+$ & 0 & - & 4 & 100,0 \\
\hline
\end{tabular}

En la tabla 4 se muestra la sobrevida según rangos de IEF. Se destaca que a mayor calificación disminuye la sobrevida. La letalidad alcanza a $100 \%$ con valores sobre 20 puntos.

Con los datos obtenidos se confeccionó una curva de probabilidad de muerte (P) según la fórmula de regresión logística $P$ : ex $(-5,84+$ $0,3768 \times \mathrm{IEF}) /(1+\exp (-5,84+0,3768 \times$ IEF)). La figura 1 muestra la curva obtenida sobre la cual se han representado en barras las tasas de letalidad observadas según rangos de IEF. No se encontró diferencia entre la letalidad esperada (P) y la observada ( $p=0,168)$.

\section{DISCUSION}

El IEF no está diseñado para asistir en decisiones referidas a pacientes individuales sino que para clasificar grupos de pacientes según su gravedad. Puesto que la severidad de una enfermedad no es susceptible de medida, se han utilizado variables fisiológicas valorables, que al otorgarles puntos, según el grado de deterioro, permiten estimar la inestabilidad fisiológica; puesto que a mayor gravedad de un paciente es mayor su pro-

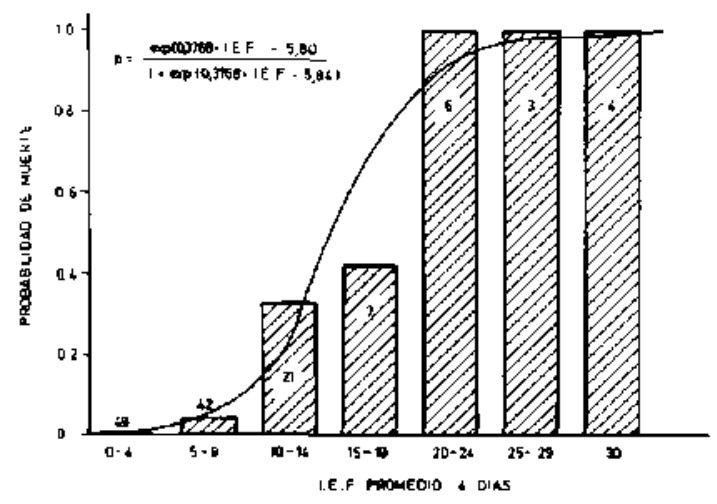

Figura I: Letalidd versus índice de estabilidad fisiológica. La curva muestra la letalidad teórica basada en análisis de regresión logística. Las barras corresponden a la mortalidad obseryada según rangos del índice de estabilidad tisiológica. Los números dentro de las bartzs son los pacientes en cada rango.

babilidad de morir, si se consiguiera establecer que a mayor inestabilidad fisiológica también aumenta la probabilidad de morir, se podría deducir que la gravedad de una enfermedad es función de los deterioros presentes en los sistemas fisiológicos separados, que es lo que mide el IEF.

De acuerdo a los datos presentados el IEF se validó como indicador de gravedad al no encontrar diferencia entre la letalidad observada según rangos de IEF y la curva teórica de probabilidad de muerte construida mediante análisís de regresión logística. Para construir dicha curva fue necesario transformar el carácter de la variable sobrevida de discreta en continua.

La validez del sistema se basa en los supuestos de que todas las variables se midan de acuerdo a las necesidades del paciente y que se proporciona terapia adecuada según esté jndicado.

La medición de menos variables fisiológicas que las necesarias podría limitar la utilidad del IEF, puesto que a deterioros no identificados no se les asigna calificación. Considerando que nuestra unidad no contaba, a la fecha del estudio, con los recursos técnicos para medir algunas de las variables que se detallan en el IEF (medición de débito cardíaco, presión pulmonar de enclavada etc.), se podría argumentar que existiría subestimación de gravedad, cosa que no sucede, ya que la curva de regresión logística se construyó a partir de datos propios, los que no incluyeron las mediciones hemodinamicas mencionadas. Este procedimiento permite afirmar que no es necesario incluir tales mediciones, ya que el criterio 
de gravedad se mantiene, tal como lo demuestra la curva obtenida.

Puesto que el sistema se valida sin estos datos, se puede plantear a futuro la posibilidad do simplificar el IEF dejando sólo variables que se midan con mayor frecuencia $y$ tengan mayor relevancia.

La utilidad más importante del sistema pre. sentado es que permite la distribución de pacientes según grupos de gravedad y de esta manera la evaluación global de una unidad de cuidado in tensivo comparando gravedad con letalidad, com plicaciones, utilización óptima de los recursos, etc. Es útil además para comparar resultados entre diversas unidades, para evaluar nuevos tratamientos y para planificar necesidades locales en cuidado intensivo pediátrico.

\section{RESUMEN}

El índice de estabilidad fisiológica (IEF) es un método para estimar la gravedad de ciertas en fermedades mediante la asignación de calificaciones a los deterioros de 34 variables fisiológicas susceptibles de ser medidas. Con el objetivo de validar el IEF, se aplicó prospectivamente a 132 pacientes de una unidad de cuidados intensivos. Los resultados mostraron diferencias significativas entre los promedios de puntuación de los sobrevivientes $(6,1$ puntos $)$ y no sobrevivientes (21 puntos) (p 0,001 ). No se encontraron diferencias entre la letalidad teóriça obtenida por medio de análisis de regresión logística y la tasa de letali. dad abservada según los rangos del puntaje IEF, aun cuando en este estudio no se incluyeron mediciones de gasto cardíaco y presión pulmonar enclavada. Se conchuye que el IEF, al correlacionarse positivamente con la letalidad, es un indicador adecuado de gravedad.

(Palabras clave: cuidados intensivos, pronóstico, indices de severidad.)

\section{REFERENCIAS}

1. Knaus W., Wagner D., Draper E., Zimmerman $J_{.}$: Severity of illness for septic patients (abstract) Crit Care Med, 1982; 10:225.

2. Pollack M., Yeh $T$., Ruttimann $U$., Holbrook $P$., Field, A: Evaluation of pediatric intensive care Crit Care Med, $1984 ; 12 ; 376-383$

3. Pollock M. Katz R., Ruttimann U., Getson P.: lmproving the outcome and efficiency of intensive care: The impact of an intensivist. Crit Care Med, $1988 ; 16: 11 \cdot 17$.

4. Rothstein P., Johnson P.: Pediatric intensive care: factors that influence outcome. Crit Care Med, 1982: 10:34-37

5. Yeh $T$., Pollack $M$. Holbrook P., Fields $A$. Ruttimonn U.: Assesmet of pediatric intensive care: application of the therapeutic intervention scoring system. Crit Care Med, 1982; 10:497-500.

6. Keene $A$, Cullen $A$.: Therapeutic intervention scoring system: update 1983. Crit Care Med, 1983, $11: 1-3$.

7. Knaus W., Zimmerman J., Wagner D., Drapner D., Lawrence D.: Apache. Acute physiology and chronic health evaluation: a physiologically based classification system. Crit Care Med 1981; 9: 591597.

8. Yeh T., Pollack M., Ruttimonn U., Holbrook P., Fields A.: Validation of a physiologic stability index for use in critically ill infants and children. Pediatr Res, 1984; 18: 445-451

9. Pollack $M$, Ruttimann $U$. Getson P., Accurate prediction of the outcome of pediatric intensive care. N Engl J Med 1987; 316 134-139.

10. Motthews D.E., Farewell V.T.: Using and underslanding medical statistics. Ed. Karger, Nueva York, 1985, pp 139-147. 